\title{
Dielectric properties of spinel ceramic $\mathrm{Fe}_{1.8} \mathrm{~V}_{1.2} \mathrm{O}_{4}$
}

\author{
LIU Na, WANG YuHang \& ZHANG LiuWan* \\ Laboratory of Advanced Materials, State Key Laboratory of Low-Dimensional Quantum Physics, Department of Physics, Tsinghua University, \\ Beijing 100084, China
}

Received June 2, 2012; accepted August 9, 2012

\begin{abstract}
Spinel vanadates possess rich physics arising from the interaction among spin, orbital and lattice degrees of freedom. We report the dielectric properties of polycrystalline $\mathrm{Fe}_{1.8} \mathrm{~V}_{1.2} \mathrm{O}_{4}$. A thermally activated dielectric relaxation appeared in low temperature due to the inhomogeneous conductivity between grains and grain boundaries. We found an artificial ferroelecticity in this sample. An abnormal frequency-independent dielectric peak appeared at room temperature when the samples were measured during warming in ambient air. However, this peak disappeared in the following cooling process. By dielectric frequency spectrum and equivalent circuit analysis in detail, we found the sample had a surface layer in warming but not in cooling process. We also confirmed that this surface layer was induced by the adsorption of water, which is responsible for the dielectric peak.
\end{abstract}

spinel, ferroelectricity, dielectric, surface layer

Citation: $\quad$ Liu N, Wang Y H, Zhang L W. Dielectric properties of spinel ceramic $\mathrm{Fe}_{1.8} \mathrm{~V}_{1.2} \mathrm{O}_{4}$. Chin Sci Bull, 2012, 57: 4707-4711, doi: 10.1007/s11434-012-5570-8

Spinel structure materials have been a hot topic due to their complicated physical properties related to the strong spinlattice coupling, and also to their highly geometrically frustrated pyrochlore structure formed by corner-sharing tetrahedra on the octahedral sites [1]. $\mathrm{FeV}_{2} \mathrm{O}_{4}$ is a normal spinel oxide in which $\mathrm{Fe}^{2+}$ and $\mathrm{V}^{3+}$ are located in the tetrahedral $\mathrm{A}$ and octahedral $\mathrm{B}$ site, respectively. With partial replacement of $\mathrm{V}$ by Fe ions, the spinel $\mathrm{Fe}_{1+x} \mathrm{~V}_{2-x} \mathrm{O}_{4}(0 \leqslant x \leqslant 2)$ family has a cation distribution of $\mathrm{Fe}^{2+}\left(\mathrm{Fe}_{x}^{3+} \mathrm{V}_{2-x}{ }^{3+}\right) \mathrm{O}_{4}$ for $0 \leqslant x \leqslant 0.35$ and $\mathrm{Fe}^{3+}\left(\mathrm{Fe}^{2+} \mathrm{Fe}_{x-1}{ }^{3+} \mathrm{V}_{2-x}{ }^{3+}\right) \mathrm{O}_{4}$ for $1 \leqslant x \leqslant 2[2]$, where the bracket indicates the $\mathrm{B}$ site. Both cation ions are magnetic and have orbital degrees of freedom. Due to the complex interactions among charge, lattice, spin, and orbital degrees of freedom, the spinel vanadates show a variety of interesting electric and magnetic properties. Takei et al. [3] discovered a magnetocapacitance effect in the polycrystalline $\mathrm{FeV}_{2} \mathrm{O}_{4}$. Nishihara et al. [4] observed a new magnetization jump on the magnetic hysteresis of $\mathrm{FeV}_{2} \mathrm{O}_{4}$ oxides. However, there is a lack of detailed dielectric investigation on the $\mathrm{Fe}_{1+x} \mathrm{~V}_{2-x} \mathrm{O}_{4}$ system. The present paper studied the temperature and fre-

*Corresponding author (email: lwzhang@tsinghua.edu.cn) quency dependent dielectric property of the $\mathrm{Fe}_{1.8} \mathrm{~V}_{1.2} \mathrm{O}_{4}$ polycrystalline. We observed a dielectric relaxation in a low temperature range and a frequency independent dielectric peak in a high temperature range.

\section{Materials and methods}

Spinel Polycrystalline $\mathrm{Fe}_{1.8} \mathrm{~V}_{1.2} \mathrm{O}_{4}$ was prepared by solidstate reaction. Thoroughly grinded stoichiometric mixture of $\mathrm{FeO}, \mathrm{Fe}_{2} \mathrm{O}_{3}$ and $\mathrm{V}_{2} \mathrm{O}_{3}$ was pressed into pellets with a thickness of about $1.5 \mathrm{~mm}$ and sealed in an evacuated silica tube with an air-pressure of $4.0 \times 10^{-5} \mathrm{~Pa}$. The pellets were sintered at $1050^{\circ} \mathrm{C}$ for $40 \mathrm{~h}$ with a regrinding in the middle. The X-ray diffraction measurement indicates that the asprepared sample is a single spinel phase with a lattice constant of $0.8346 \mathrm{~nm}$ which is consistent with literature [5]. Scanning electron microscope (SEM) images show that the sample is porous with an average grain size of several micrometers (inset of Figure 2(c)). Silver gel was pasted on the opposite sides of the pellet as ohmic contact electrodes with a diameter of $7 \mathrm{~mm}$. The dielectric properties were measured by a QuadTech 1730 LCR Digibridge and an im- 
pendence analyzer (Novocontrol) over a frequency range from $1 \mathrm{~Hz}$ to $10 \mathrm{MHz}$ and a temperature range from 77 to $360 \mathrm{~K}$ at an oscillation voltage of $10 \mathrm{mV}$. The measurements were performed in ambient air or in vacuum of a closed cycle refrigerator.

\section{Results and discussion}

Figure 1(a) and (b) show the temperature $(T)$ dependent real $\left(\varepsilon^{\prime}(T)\right)$ and imaginary part $\left(\varepsilon^{\prime \prime}(T)\right)$ of dielectric constant at different frequencies in the temperature range from 77 to $150 \mathrm{~K}$. Two $\varepsilon^{\prime}(T)$ plateaus appear at high and low temperatures, respectively. Between the two plateaus, an approximate 1000-fold drop is observed, accompanied by a peak on the corresponding imaginary curve, which was widely observed in the transition metal oxides. The increase in measuring frequency $f$ shifts the peak temperature $T_{\mathrm{p}}$ to a higher temperature, indicating a thermally activated Debye-like relaxation behavior. The Arrhenius plot of $\ln f$ vs. $1 / T_{\mathrm{p}}$ shows a linear behavior (inset of Figure 1(a)) with activation energy $E_{\mathrm{g}}$ of $107.5 \mathrm{meV}$. The dielectric plateaus as well as the great drop in between at low temperatures were widely observed in transition metal oxides [6-9], which is usually ascribed to the Maxwell-Wagner type dielectric relaxation due to the electric conduction inhomogeneity in the matrix, at grain boundary (GB) or at the electrode-sample interface (EI) [10,11]. In our case, the electrode contacts are ohmic. Therefore, the dielectric relaxation may come from the conductivity difference between the grains and grain boundaries.

Figure 1(c) presents the complex impedance (CI) of the sample measured in ambient air at $130 \mathrm{~K}$. The intercept of the arc to the horizontal axis at high frequency zone is not zero as seen from the expended view in the upper left inset. The CI spectra were analyzed using an equivalent circuit (EC) model. Empirically, the phenomenological constant phase element (CPE), which is a capacitive element with non-Debye behavior, is used to replace the ideal capacitor with Debye behavior due to the imperfect blocking characteristics of GBs and EIs [12-14]. Our EC is composed of a set of parallel ideal resistor $\left(R_{\mathrm{g}}\right)$ and ideal capacitor $\left(C_{\mathrm{g}}\right)$ circuit (RC-circuit) for grains and a set of parallel ideal resistor $\left(R_{\mathrm{gb}}\right)$ and non-ideal CPE circuit (R-CPE) for grain boundaries as schematically shown in the upper right inset of Figure 1(c). The corresponding equation for the complex impedance is $R(f)=\sum_{j}\left[R_{j}^{-1}+C_{m j}(i 2 \pi f)^{\alpha}\right]^{-1}$, where $j$ rep-

resents grains and GBs, $C_{m}$ is the capacitance that is modified from the ideal capacitance, and $\alpha$ is used to describe the width of the material property distribution. $\alpha$ lies between zero and unity and is set to unity for the ideal capacitance of grains. The best fits for the experimental data are indicated by the solid line in Figure 1(c), and the used parameters are
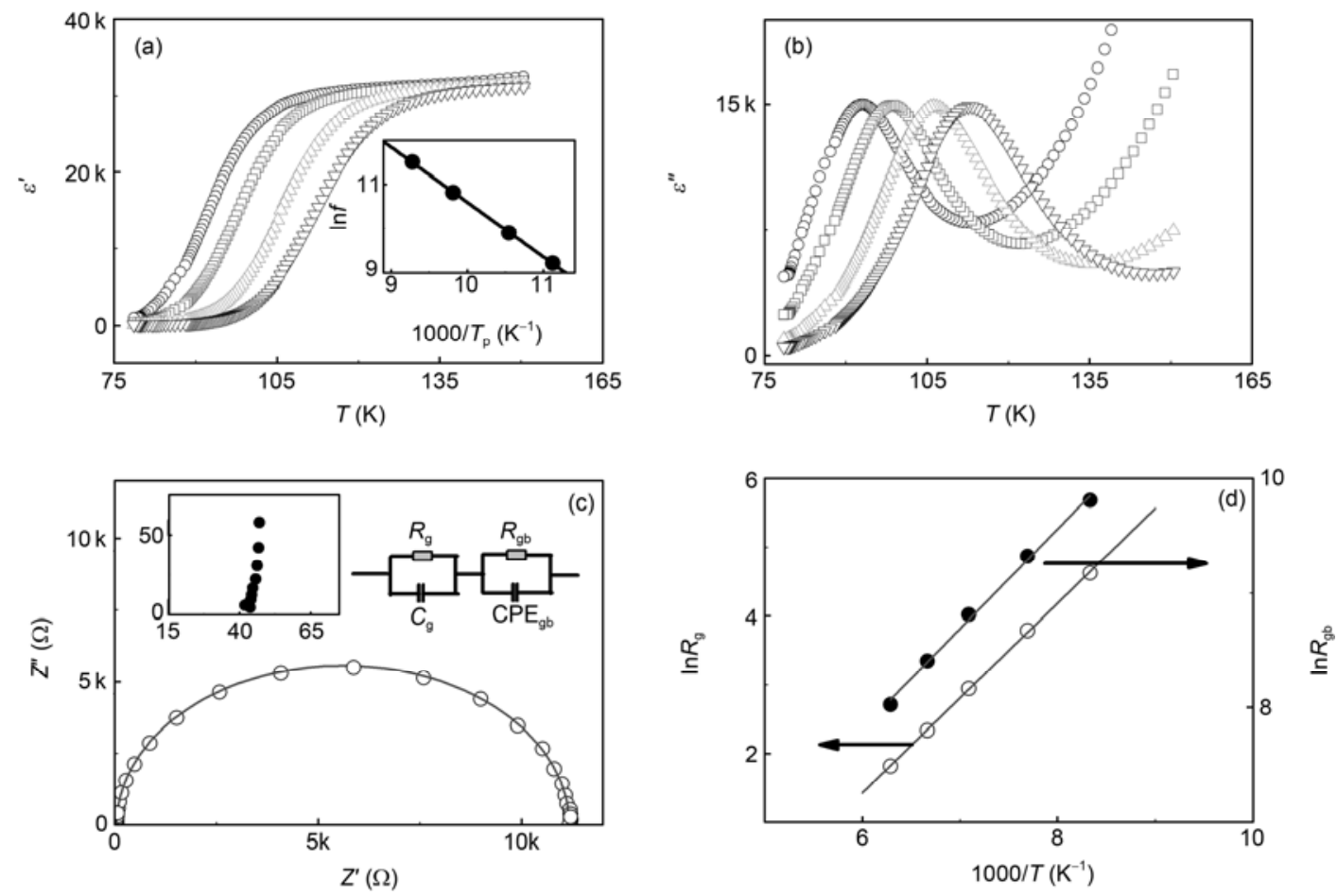

Figure 1 Real part (a) and imaginary part (b) of dielectric constant as a function of temperature from 77 to $150 \mathrm{~K}$ at measuring frequency: $10 \mathrm{kHz}$ (circles), $20 \mathrm{kHz}$ (squares), $50 \mathrm{kHz}$ (up triangles) and $100 \mathrm{kHz}$ (down triangles). Inset of (a): Arrhenius plot of lnf vs. 1000/T . (c) Complex impedance at $130 \mathrm{~K}$. Insets: expanded view of high frequency zone (upper left); and equivalent circuit (upper right). (d) Arrhenius plots of fitted resistance of grains $\left(R_{\mathrm{g}}\right)$ and grain boundaries $\left(R_{\mathrm{gb}}\right)$. 
summarized in Table 1. It is noticed that at all temperatures $\alpha \approx 1$, indicating that the CPE for grain boundaries is near to ideal capacitance. Both $R_{\mathrm{g}}$ and $R_{\mathrm{gb}}$ satisfy the relation of $R_{j}=R_{j 0} \exp \left(E_{j} / k T\right)$, where $k$ Boltzmann constant, $R_{j 0}$ preexponential factor, and $E_{j}$ activation energy. The activation energy is $118.9 \mathrm{meV}$ for grain, and $74.2 \mathrm{meV}$ for grain boundary obtained from the linear fittings of $\ln R_{i}$ vs. $1 / T$ (Figure 1(d)). The fitted activation energy of grain is in good agreement with the dielectric relaxation activation energy of $107.5 \mathrm{meV}$ mentioned above. This is not incidental because according to the EC in Figure 1(c) if $\alpha$ is set to 1 , the dielectric relaxation time $[15,16] \tau \sim R_{\mathrm{g}}(T) C_{m \mathrm{gb}}$.

The dielectric constant in the temperature range of 200 $340 \mathrm{~K}$ at different frequencies measured in ambient air is displayed in Figure 2(a) for warming and Figure 2(b) for cooling. The warming curve features a broad peak near room temperature. The peak temperature is independent of measuring frequencies. However, no peak appears in the

Table 1 Resistances and capacitance of grain and grain boundary at low temperatures

\begin{tabular}{cccccc}
\hline$T(\mathrm{~K})$ & $R_{\mathrm{g}}(\Omega)$ & $C_{\mathrm{g}}(\mathrm{pF})$ & $R_{\mathrm{gb}}(\Omega)$ & $C_{m \mathrm{gb}}(\mathrm{nF})$ & $\alpha_{\mathrm{gb}}$ \\
\hline 120 & 101.77 & 19.941 & 18170 & 6.2446 & 0.99740 \\
130 & 44.12 & 29.522 & 11176 & 6.9734 & 0.98704 \\
141 & 18.958 & 25.667 & 6711.1 & 7.0358 & 0.98735 \\
150 & 10.348 & 2.68 & 4475.7 & 6.9928 & 0.98879 \\
159 & 6.1691 & 0.298 & 3060.7 & 7.1284 & 0.98864 \\
\hline
\end{tabular}

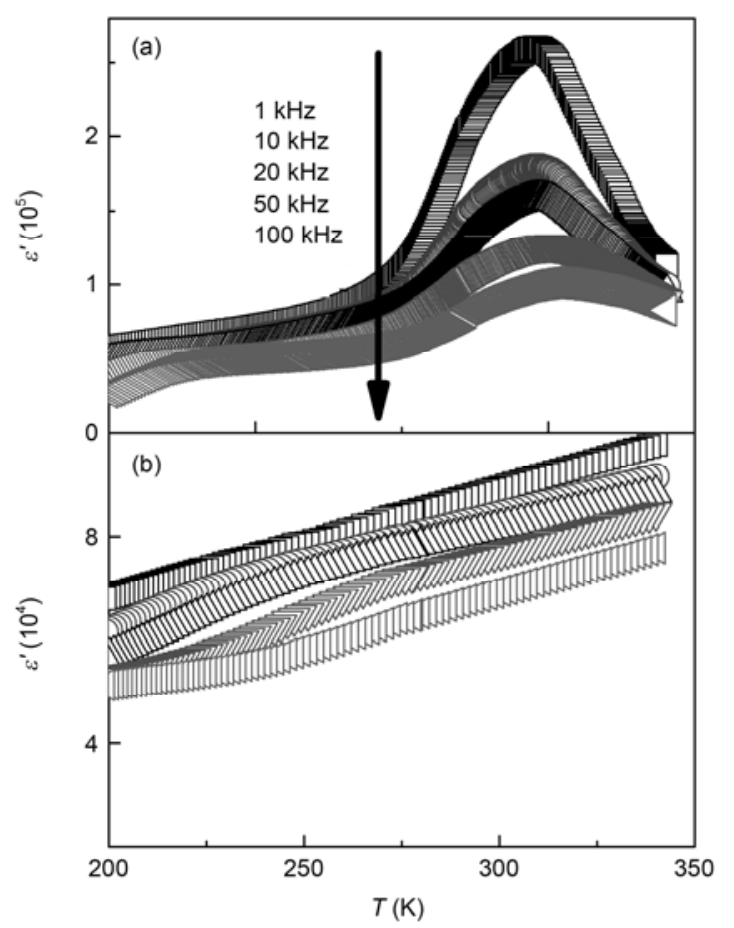

following cooling run (Figure 2(b)). Continuous thermal cycling in the same temperature range suppresses the peak amplitude, and shifts the peak temperature to a little higher temperature. But the peak was not observed during cooling. The existence of a peak in the real part of the dielectric constant was reminded of the ferroelectric phase transition [17-20]. The cooperation of the localized positive and negative carriers as well as the Maxwell-Wagner-type relaxation can also generate a dielectric peak [21-23]. However, the frequency independence of the peak position as well as the absence of the dielectric peak in the cooling process in our experiments excludes these possibilities. Similar dielectric peaks were observed in Li-doped, Be-doped and $\mathrm{Mg}$ doped $\mathrm{ZnO}$ ceramics [24,25], which was attributed to the ferroelectric phase transition by the authors. Recently, Soukiassian et al. [26] pointed out that the observed dielectric peak in the doped $\mathrm{ZnO}$ bulk and thin films was an artifact, originating from the presence of water in the measurement system. Nevertheless, how the water in ambient air caused the dielectric peak remains unclear.

To find out the water effects on the dielectric constant, in Figure 2(c) and (d) we recorded the change in dielectric constant at room temperature during the following consecutive processes: (1) we dried a test sample at $200^{\circ} \mathrm{C}$ for 20 min with the final apparent dielectric constant of about $4.8 \times 10^{4}$; (2) we exposed the sample to the water saturated air for $30 \mathrm{~min}$; (3) we moved the sample to the vacuum chamber and started pumping. As seen from Figure 2(c),

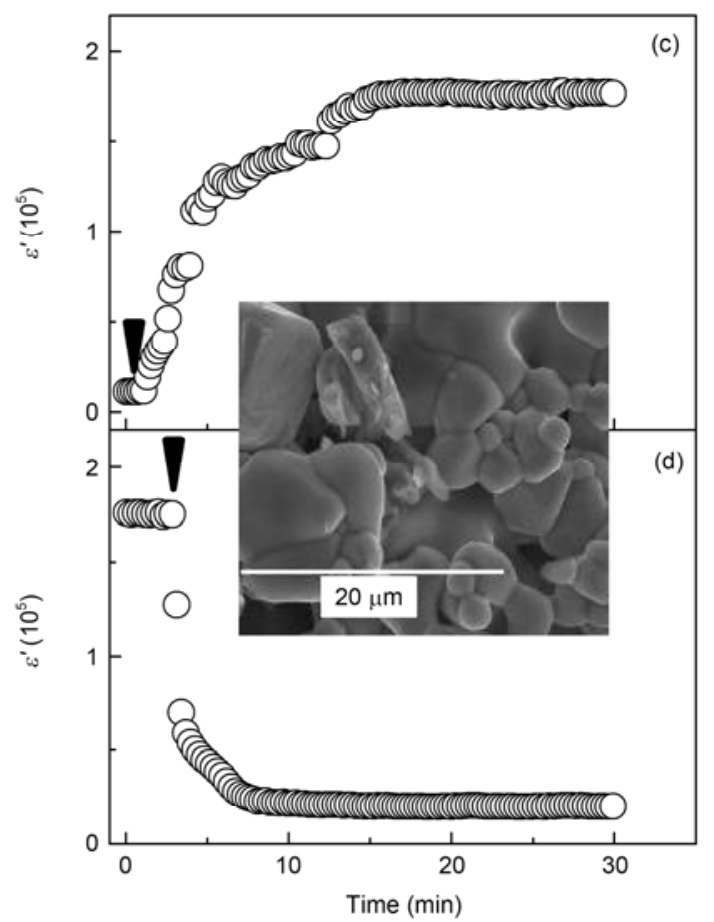

Figure 2 Temperature dependent dielectric constant in ambient air during warming (a) and cooling (b) process. Variation of the dielectric constant with time upon exposing the sample to water saturated air (c), and during the subsequent pumping in vacuum (d). The measuring frequency is $1 \mathrm{kHz}$. The arrows indicate the respective starting time. Inset of (c): SEM image of the sample. 
upon exposing the dried sample to the wetted air, the apparent dielectric constant was rapidly increased from $1.1 \times 10^{4}$ to an approximate equilibrium value of $1.8 \times 10^{5}$ in less than $20 \mathrm{~min}$. With pumping the dielectric constant of the same sample dropped quickly (Figure 2(d)) to $2 \times 10^{4}$ within $10 \mathrm{~min}$. Soon after the first or third process the sample was thermally cycled in vacuum in the same temperature range as in Figure 2(a) and (b). The warming and cooling curves similar to Figure 2(b) (not shown here) were overlapping without any dielectric peak. These results indicate that the appearance and disappearance of the dielectric peak observed in ambient air are related to the water adsorption and desorption in the sample.

To further clarify the mechanism underlying the dielectric peak in Figure 2(a), the CI spectra were measured in the temperature range from 200 to $365 \mathrm{~K}$ in ambient air, and analyzed with EC model as typically shown in Figure 3 at $293 \mathrm{~K}$. In the cooling process, the CI spectra at each temperature can be well fitted by one simple parallel R-CPE circuit $\left(R_{\mathrm{gbc}}, \mathrm{CPE}_{\mathrm{gbc}}\right.$ in Figure 3(b)). This is expected be-

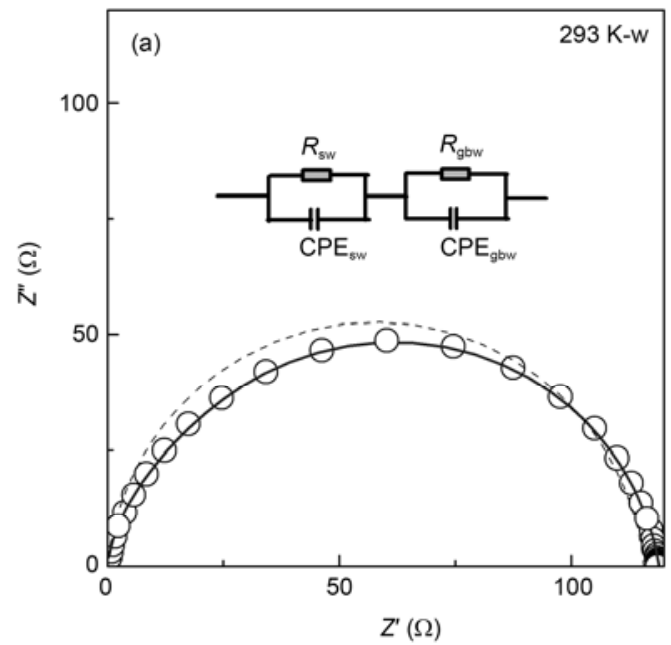

cause in the measured temperature range the equivalent resistance of the grain should be negligible inferred from Table 1. The CI spectra are controlled by the grain boundary. On the contrary, the best fitting to the CI spectra during warming, especially at temperatures around the dielectric peak, can only be obtained by two R-CPE circuits connected in series $\left(R_{\mathrm{gbw}}\right.$ and $\mathrm{CPE}_{\mathrm{gbw}}, R_{\mathrm{sw}}$ and $\mathrm{CPE}_{\mathrm{sw}}$ in Figure 3(a)). All the fitting parameters are listed in Table 2. In the upper inset of Figure 3(b), the fitted $R_{\mathrm{gbw}}$ and $R_{\mathrm{gbc}}$ were plotted logarithmically as a function of $1 / T$. The apparent linear correlations indicate that both $R_{\mathrm{gbw}}$ and $R_{\mathrm{gbc}}$ satisfy Arrehenius equations with activation energy of $121.6 \mathrm{meV}$ and $123.04 \mathrm{meV}$, respectively. The almost same values of the activation energy imply that the parallel circuits of $R_{\mathrm{gbw}}{ }^{-}$ $\mathrm{CPE}_{\mathrm{gbw}}$ and $R_{\mathrm{gbc}}-C_{\mathrm{gbc}}$ represent the grain boundaries.

Let us focus on the source of another equivalent parallel circuit of $R_{\mathrm{sw}}$ and $\mathrm{CPE}_{\mathrm{sw}}$. As already revealed in Figure 2(c), the rapid response of the apparent dielectric constant to the wetted air suggested that the absorbed water may go into the EIs or penetrate into the surface of the grain boundaries due

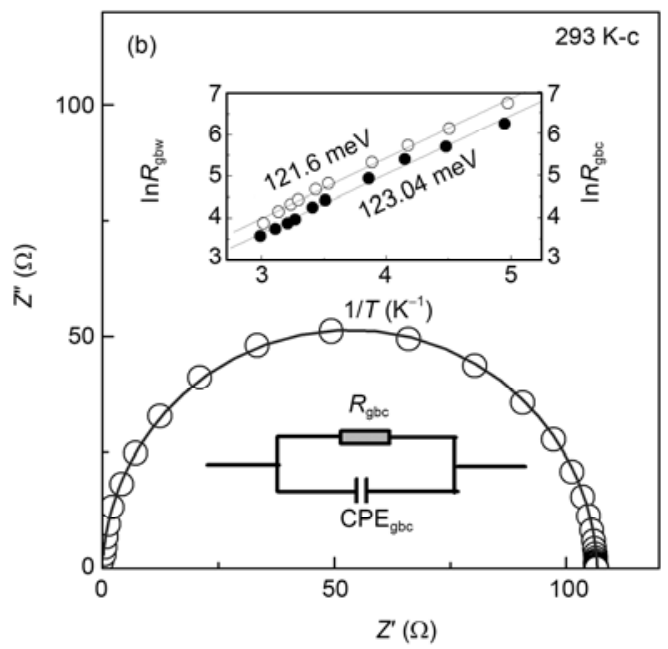

Figure 3 Complex impedance at $293 \mathrm{~K}$ in ambient air in the warming (a) and cooling (b) process. The best fittings with the equivalent circuits shown in the insets are plotted by solid lines. For comparison, the fitting result with one parallel R-CPE circuit is also shown in (a) by dashed line. Upper inset of (b): Arrhenius plots and the linear fittings of the grain boundary resistance during warming (open dots) and cooling (filled dots) process in ambient air. The values of the activation energy are indicated.

Table 2 Fitting parameters of equivalent circuits in the temperature range of 200-364 K

\begin{tabular}{|c|c|c|c|c|c|c|c|c|c|}
\hline$T(\mathrm{~K})$ & $R_{\mathrm{sw}}(\Omega)$ & $C_{m \mathrm{sw}}(\mathrm{nF})$ & $\alpha_{\mathrm{sw}}$ & $R_{\mathrm{gbw}}(\Omega)$ & $C_{\text {mgbw }}(\mathrm{nF})$ & $\alpha_{\mathrm{gbw}}$ & $R_{\mathrm{gbc}}(\Omega)$ & $C_{m \mathrm{gbc}}(\mathrm{nF})$ & $\alpha_{\mathrm{gbc}}$ \\
\hline 364 & - & - & - & 26.795 & 18.618 & 0.93632 & 26.795 & 18.618 & 0.93632 \\
\hline 335 & 17.598 & 38.038 & 0.86888 & 35.612 & 25.322 & 0.97134 & 48.211 & 11.744 & 0.96186 \\
\hline 322 & 28.788 & 85.0593 & 0.86054 & 42.21 & 30.572 & 0.97968 & 63.25 & 10.992 & 0.96543 \\
\hline 312 & 37.779 & 91.864 & 0.86751 & 48.507 & 34.317 & 0.98461 & 75.544 & 10.334 & 0.96897 \\
\hline 306 & 41.57 & 99.699 & 0.86603 & 53.021 & 42.631 & 0.97492 & 83.444 & 10.061 & 0.97052 \\
\hline 293 & 48.197 & 190.15 & 0.84974 & 70.721 & 85.985 & 0.94905 & 106.53 & 9.0949 & 0.97659 \\
\hline 259 & 63.093 & 92.412 & 0.87886 & 140.72 & 59.511 & 0.93435 & 202.05 & 7.865 & 0.98483 \\
\hline 241 & 63.907 & 32.699 & 0.94575 & 223.67 & 28.406 & 0.95313 & 303.55 & 7.3516 & 0.98889 \\
\hline 223 & 122.98 & 19.779 & 0.96334 & 301.81 & 16.449 & 0.99305 & 455.2 & 6.8842 & 0.99296 \\
\hline 202 & 199.55 & 17.708 & 0.97383 & 523.83 & 14.094 & 0.99227 & 833.5 & 6.6035 & 0.99554 \\
\hline
\end{tabular}


to the porous nature of our sample. Usually, the value of CPE for EIs is much larger than that for grain boundaries. From Table 2, it is clearly seen that at the same temperature, the fitted resistance of grain boundary during cooling $\left(R_{\mathrm{gbc}}\right)$ is larger than the counterpart during warming $\left(R_{\mathrm{gbw}}\right)$, and $\mathrm{CPE}_{\mathrm{sw}}$ and $\mathrm{CPE}_{\mathrm{gbw}}$ almost have the same order of amplitude. Therefore it is most likely that the $R_{\mathrm{sw}}$ and $\mathrm{CPE}_{\mathrm{sw}}$ parallel circuit during warming represents the contribution from the surface of GBs instead of EIs.

According to the above discussion, the dielectric peak appearing during warming in the ambient air can be explained as follows. When the sample is cooled in ambient air across the freezing point of water, the moisture within the air condenses into ice around the surface. During the following warming through the melting point of ice, small drops of water cover the sample and the water saturated air quickly flows into the sample by the connected meandering micropores. The moisture wets the surface and partially penetrates into the grain boundary owing to the disordering and dislocations. As a result, the original grain boundary forms a core-shell structure, corresponding to two sets of parallel R-CPE circuits. Because the dielectric constant of water is larger than that of our sample, adsorption of water in the partial grain boundary increased the dielectric constant. At higher temperatures the water starts to evaporate out of the grain boundary. Consequently the dielectric constant decreases, and a peak occurs as shown in Figure 2(a). However, in the subsequent cooling process the moisture in the ambient air condenses into ice on the sample surface so quickly that the water has no enough time to diffuse into the grain boundary. Therefore, no dielectric peak was observed during cooling (Figure 2(b)).

\section{Conclusions}

In summary, the dielectric properties of polycrystalline $\mathrm{Fe}_{1.8} \mathrm{~V}_{1.2} \mathrm{O}_{4}$ ceramics have been studied. A Maxwell-Wagner like dielectric relaxation appeared in the temperature range from 77 to $150 \mathrm{~K}$ due to the inhomogeneity in conductivity between grains and grain boundaries. In the high temperature range of $200-360 \mathrm{~K}$ an anomalous frequency independent dielectric peak at about room temperature is observed in the ambient warming process, but the peak disappears during the subsequent cooling. The adsorption and desorption of water in the surface of the grain boundaries are responsible for the appearance and disappearance of the dielectric peak.

This work was supported by the National Basic Research Program of China (2011CB921904, 2012CB927402), the National Natural Science Foundation of China (11074142, 11021464), Key Project of Chinese Ministry of Education (309003) and Tsinghua TNList Cross-discipline Foundation.
1 Xu Z G, Cheng F X, Zhou B, et al. Combustion synthesis and magnetic investigation of nanosized $\mathrm{CoFe}_{2} \mathrm{O}_{4}$. Chin Sci Bull, 2001, 46: 384-387

2 Abe M, Kawachi M, Nomura S. Mössbauer study of the $\mathrm{FeV}_{2} \mathrm{O}_{4}$ $\mathrm{Fe}_{3} \mathrm{O}_{4}$ system. J Solid State Chem, 1974, 10: 351-356

3 Takei H, Suzuki T. Nonvolatile memory effect of capacitance in polycrystalline spinel vanadate. Appl Phys Lett, 2007, 91: 072506

4 Nishihara S, Doi W, Ishibashi H, et al. Appearance of magnetization jumps in magnetic hysteresis curves in spinel oxide $\mathrm{FeV}_{2} \mathrm{O}_{4}$. J Appl Phys, 2010, 107: 09A504

5 Rogers D B, Arnott R J, Wold A, et al. The preparation and properties of some vanadium spinels. J Phys Chem Solids, 1963, 24: 347-360

6 Zhang L, Chen X M. Dielectric relaxation in $\mathrm{LuFeO}_{3}$ ceramics. Solid State Commun, 2009, 149: 1317-1321

7 Ramirez A P, Subramanian M A, Gardel M, et al. Giant dielectric constant response in a copper-titanate. Solid State Commun, 2000, 115: 217-220

8 Lunkenheimer P, Krohns S, Riegg S, et al. Colossal dielectric constants in transition-metal oxides. Europ Phys J, 2009, 180: 61-89

$9 \mathrm{Xu} \mathrm{Z}$, Zheng S G, Yue Z X, et al. Research on effects of hydrostatic pressure on the dielectric property of $\mathrm{Pb}\left(\mathrm{Zn}_{1 / 3} \mathrm{Nb}_{2 / 3}\right) \mathrm{O}_{3}-\mathrm{BaTiO}_{3} \mathrm{PbTiO}_{3}$ relaxor ferroelectric ceramics. Chin Sci Bull, 2002, 47: 513-518

10 Adams T B, Sinclair D C, West A R. Giant barrier layer capacitance effects in $\mathrm{CaCu}_{3} \mathrm{Ti}_{4} \mathrm{O}_{12}$ ceramics. Adv Mater, 2002, 14: 1321-1323

11 Wang C C, Cui Y M, Xie G L, et al. Phase separation in $\mathrm{La}_{2} \mathrm{CuO}_{4+y}$ ceramics probed by dielectric measurements. Phys Rev B, 2005, 72: 064513

12 Schmidt R, Eerenstein W, Winiecki T, et al. Impedance spectroscopy of epitaxial multiferroic thin films. Phys Rev B, 2007, 75: 245111

13 Meher K R S P, Varma K B R. Colossal dielectric behavior of semiconducting $\mathrm{Sr}_{2} \mathrm{TiMnO}_{6}$ ceramics. J Appl Phys, 2009, 105: 034113

14 Liu S H, Huang J C A, Qi X D, et al. Structural transformation and charge transfer induced ferroelectricity and magnetism in annealed $\mathrm{YMnO}_{3}$. AIP Adv, 2011, 1: 032173

15 Wang C C, Zhang L W. Surface-layer effect in CaCuTiO. Appl Phys Lett, 2006, 88: 042906

16 Cao G H, Feng L X, Wang C. Grain-boundary and subgrain-boundary effects on the dielectric properties of $\mathrm{CaCu}_{3} \mathrm{Ti}_{4} \mathrm{O}_{12}$ ceramics. J Phys D: Appl Phys, 2007, 40: 2899-2905

17 Fan H Q, Xu Z, Zhang L Y, et al. Dielectric behavior of poled complex perovskite relaxor ferroelectrics. Chin Sci Bull, 1997, 42: 169-172

18 Wang J, Li M Y, Liu X L, et al. Synthesis and ferroelectric properties of $\mathrm{Nd}$ doped multiferroic $\mathrm{BiFeO}_{3}$ nanotubes. Chin Sci Bull, 2010, 55: 1594-1597

19 Dai Z H, Yao X, Xu Z, et al. Properties of PbLa(Zr,Sn,Ti)O $\mathrm{O}_{3}$ ceramics near ferroelectric-antiferroelectric phase boundary. Chin Sci Bull, 2006, 51: 1000-1004

$20 \mathrm{Wu} N \mathrm{~N}$, Song X M, Hou Y D, et al. Relaxor behavior of $(1-x)$ $\mathrm{Pb}\left(\mathrm{Mg}_{1 / 3} \mathrm{Nb}_{2 / 3}\right) \mathrm{O}_{3}-x \mathrm{PbTiO}_{3}$ ceramics. Chin Sci Bull, 2009, 54: 12671274

21 Wang C C, Dou S X. Pseudo-relaxor behaviour induced by MaxwellWagner relaxation. Solid State Commun, 2009, 149: 2017-2020

22 Wang $\mathrm{C} \mathrm{C}$, Zhang L W. Oxygen-vacancy-related dielectric anomaly in $\mathrm{CaCu}_{3} \mathrm{Ti}_{4} \mathrm{O}_{12}$ : Post-sintering annealing studies. Phys Rev B, 2006, 74: 024106

23 Ke S M, Huang H T, Fan H Q. Relaxor behavior in $\mathrm{CaCu}_{3} \mathrm{Ti}_{4} \mathrm{O}_{12}$ ceramics. Appl Phys Lett, 2006, 89: 182904

24 Onodera A. Novel ferroelectricity in II-VI semiconductor ZnO. Ferroelectrics, 2002, 267: 131-137

25 Onodera A, Tamaki N, Kawamura Y, et al. Dielectric activity and ferroelectricity in piezoelectric semiconductor Li-doped ZnO. Jpn J Appl Phys, 1996, 35: 5160-5162

26 Soukiassian A, Tagantsev A, Setter N. Anomalous dielectric peak in $\mathrm{Mg}$ and Li doped $\mathrm{ZnO}$ ceramics and thin films. Appl Phys Lett, 2010, 97: 192903

Open Access This article is distributed under the terms of the Creative Commons Attribution License which permits any use, distribution, and reproduction in any medium, provided the original author(s) and source are credited. 